\title{
Ab Initio Study of the Elastic and Mechanical Properties of B19 TiAl
}

\author{
Yufeng Wen ${ }^{1,2, *}$, Long Wang ${ }^{1,2}$, Huilong Liu ${ }^{1,2}$ and Lin Song ${ }^{3}$ \\ 1 Institute of Atomic and Molecular Physics and Functional Materials, Jinggangshan University, \\ Ji'an 343000, China; 18397965571@163.com (L.W.); 15979695819@163.com (H.L.) \\ 2 School of Mathematical Sciences and Physics, Jinggangshan University, Ji'an 343000, China \\ 3 State Key Laboratory of Solidification Processing, Northwestern Polytechnical University, \\ Xi'an 710072, China; songlin@nwpu.edu.cn \\ * Correspondence: jgsuwyf@sina.com; Tel.: +86-796-8100-488
}

Academic Editor: Helmut Cölfen

Received: 27 November 2016; Accepted: 1 February 2017; Published: 15 February 2017

\begin{abstract}
A theoretical study of the structural, elastic, and mechanical properties of B19 TiAl intermetallic compound with orthorhombic structure has been carried out by using ab initio density functional theory calculations based on the projector augmented wave and the generalized gradient approximation. The optimized structure parameters are found to be in agreement with the available experimental data. The orthorhombic B19 structure of TiAl is found to be mechanically stable at ground state in terms of formation energy and single crystal elastic constants. Additionally, the polycrystalline bulk, shear and Young's moduli, Poisson's ratio, Cauchy pressure, and anisotropy factors are obtained from the single crystal elastic constants. At ground state, the B19 TiAl is found to not only have intrinsic brittleness in terms of Pugh's ratio, Poisson's ratio, and Cauchy pressure, but also exhibit elastic anisotropy in terms of elastic anisotropy factors and orientation dependence of Young's modulus.
\end{abstract}

Keywords: B19 TiAl; elastic property; mechanical property; ab initio

\section{Introduction}

$\gamma$-TiAl-based intermetallics with low densities and superior high-temperature properties are extensively recognized as promising light-weight high-temperature structural materials for aerospace and automotive applications [1]. However, a broad industrial implementation of these intermetallics is severely inhibited by their room-temperature brittleness and limited hot workability. Alloying and heat treatments are effective ways to overcome the brittleness and to improve the hot workability. The usage of these techniques can lead to the formations of several lower symmetry ordered phases in the microstructure [1-3]. Among lower symmetry phases, the orthorhombic B19 phase is commonly observed in $\gamma$-TiAl-based intermetallics. Abe et al. [2] reported the formation of B19 TiAl phase in metastable $\alpha_{2}$ ( $\mathrm{D}_{19}$ structure) phase after rapid cooling in a Ti-48Al alloy. Tanimura et al. [4] observed the appearance of B19 TiAl phase during the structural change from $\alpha$ (A3 structure) to $\alpha_{2}+\gamma\left(\mathrm{L} 1_{0}\right.$ structure) lamellar structures in a Ti-40Al alloy. Ducher et al. [5] observed the variants of B19 TiAl phase to form in Fe containing $\gamma$ matrix in Ti-48Al-2Cr-2Nb alloy. Appel et al. [3] made a careful observation of $\mathrm{B} 19$ phase in Ti-(40-44)Al-8.5Nb alloys and admitted the B19 phase to be a transitional phase between the cubic $\beta$ (A2 structure) and/or $\beta_{\mathrm{O}}$ (B2 structure) phase and the orthorhombic $\mathrm{O}-\mathrm{Ti}_{2} \mathrm{AlNb}$ phase. Additionally, the occurrence of $\mathrm{B} 19$ phase was observed by Schmoelzer et al. [6] in Ti-45Al-3Mo-0.1B alloy and by Song et al. [7] in Ti-45Al-8.5Nb-0.2W-0.2B-0.02Y alloy, which facilitates the precipitation of $\gamma$ phase from $\alpha_{2}$ phase. The existence of B19 phase has a significant 
impact on the mechanical properties of the intermetallics [3,8-10]. Therefore, it is necessary to study the mechanical properties of B19 phase to better understand its role in $\gamma$-TiAl-based intermetallics.

Elastic constants are fundamental and indispensable to describe the mechanical properties of materials. They can be used to determine the bulk modulus $B$, shear modulus $G$, Young's modulus $E$, Poisson's ratio $v$, Cauchy pressure, and elastic anisotropy factor of materials. It is very obvious that the elastic constants play an important role in determining the strength of the materials. Furthermore, Pugh [11] has introduced the ratio between the shear modulus and the bulk modulus $(G / B)$ to distinguish the ductile/brittle behaviors of materials. A low (high) $G / B$ value is correlated with the ductility (brittleness) of the materials. The critical value of the brittle-to-ductile transition was observed to be 0.5 . Pettifor [12] has introduced the Cauchy pressure to describe the angular character of atomic bonding related to the ductile/brittle characteristics of the materials. He suggested that a larger positive Cauchy pressure corresponds to a ductile material with more metallic bonds, whereas larger negative values indicate a brittle behavior with a more angular character of bonds. Additionally, Frantsevich et al. [13] have distinguished the ductility/brittleness of the materials in terms of Poisson's ratio. The ductile materials have a larger Poisson's ratio than $1 / 3$. Besides, the elastic constants can be also used to judge the mechanical stability of the materials with different crystal structures [14]. Therefore, a comprehensive analysis of elastic properties is necessary for better understanding the mechanical properties of the materials.

With the rapid development of computer technology and computational science, the ab initio calculation method has become an effective technique to study the properties of materials. In recent years, the ab initio calculation method has been successfully used in investigating the elastic and mechanical properties of some Ti-Al intermetallic compounds [15-22]. Besides, Nguyen-Manh and Pettifor $[23,24]$ have successfully studied the structural phase transitions related to the B19 phase in $\gamma$-TiAl-based intermetallics by using ab initio calculations. Most recently, Holec et al. [25] took the ab initio calculation method to study the preferential site occupations of ternary elements in B19 TiAl. To our knowledge, the elastic and mechanical properties of B19 TiAl have not been reported in the literature. Motivated by the interest in the elastic and mechanical properties of B19 TiAl, in this paper we first determine its single crystal elastic constants by using ab initio calculations. Subsequently, we further investigate the polycrystalline elastic and mechanical properties of B19 TiAl. The rest of the arrangements are as follows: Section 2 describes the computational methodology in brief. Section 3 presents the single crystal elastic constants, polycrystalline elastic moduli, and elastic anisotropy along with detailed discussions. Finally, the conclusions are drawn in Section 4.

\section{Computational Methodology}

\subsection{Computational Details}

$\mathrm{Ab}$ initio calculations on orthorhombic B19 TiAl were performed using the density functional theory (DFT) based on the projector augmented wave [26] and the generalized gradient approximations (GGA) parameterized by Perdew, Burke, and Ernzerhof (PBE) [27,28], as implemented in the Vienna $\mathrm{Ab}$ initio Simulation Package (VASP) [29-31]. The configurations Ti $3 s^{2} 3 \mathrm{p}^{6} 3 \mathrm{~d}^{2} 4 \mathrm{~s}^{2}$ and $\mathrm{Al} 3 \mathrm{~s}^{2} 3 \mathrm{p}^{1}$ were treated as the valence electrons. An energy cutoff of $600 \mathrm{eV}$ was used for the expansion of electronic wave functions. A convergence threshold of $10^{-6} \mathrm{eV}$ was used for the total energy of the electronic self-consistency. A K-point mesh of $11 \times 19 \times 11$ was generated using the Monkhorst-Pack scheme [32] for numerical integrations over the Brillouin zone of B19 TiAl. Before the calculations of elastic constants, the structure was fully relaxed with respect to the volume, shape, and internal atomic positions until the atomic forces were less than $0.01 \mathrm{eV} / \AA$ for unit cell.

\subsection{Calculations of Elastic Constants}

An orthorhombic crystal has nine independent second-order elastic constants $C_{11}, C_{22}, C_{33}, C_{12}$, $C_{13}, C_{23}, C_{44}, C_{55}$, and $C_{66}$. We take two different ab initio techniques to determine the nine single 
crystal elastic constants of orthorhombic B19 TiAl. One is the energy density method (EDM), in which the elastic constants are defined as the second derivatives of the strain energy per volume with respect to the selected strain [33-35]. After imposing a small Lagrangian strain $\eta$ on the perfect unit cell with the equilibrium structural parameters, the strain energy density $\left(\Delta E / V_{0}\right)$ can be Taylor expanded in powers of the strain tensor as

$$
\frac{\Delta E}{V_{0}}=\frac{1}{2 !} \sum_{i, j=1}^{6} C_{i j} \eta_{i} \eta_{j}+O\left(\eta^{3}\right)
$$

where $V_{0}$ is the volume of the perfect unit cell, and $C_{i j}$ represents the second-order elastic constants in the Voigt notation, which replaces $x x, y y, z z, y z, x z$, and $x y$ by $1,2,3,4,5$, and 6 , respectively. To obtain the second-order elastic constants of B19 TiAl, we selected nine different strain modes given in Table 1 to impose on the perfect unit cell with the equilibrium structural parameters. To minimize the errors coming from higher-order terms, eleven strain energy data were calculated by varying the value of tensor parameter $\delta$ from -0.015 to 0.015 in steps of 0.003 for each strain mode. The elastic constants $C_{i j}$ can derive from the resulting variation in the strain energy density. Another is the strain-stress method (SSM) embedded in the VASP, in which the elastic constants are defined as the first derivatives of the stresses with respect to the strain tensor [36]. The elastic tensor is determined by performing six finite distortions of the lattice and deriving the elastic constants from the strain-stress relationship. The elastic tensor is calculated both for rigid ions, as well, as allowing for relaxation of the ions. The ionic contributions are determined by inverting the ionic Hessian matrix and multiplying it with the internal strain tensor $[37,38]$. The final elastic constants include both the contributions for distortions with rigid ions and the contributions from the ionic relaxations.

Table 1. Selected strains $(\eta)$ and the corresponding strain energy densities $\left(\Delta E / V_{0}\right)$ for orthorhombic B19 TiAl [34].

\begin{tabular}{ccc}
\hline No. & $\eta$ & $\Delta E / V_{\mathbf{0}}$ \\
\hline D1 & $(\delta, 0,0,0,0,0)$ & $1 / 2 C_{11} \delta^{2}$ \\
D2 & $(0, \delta, 0,0,0,0)$ & $1 / 2 C_{22} \delta^{2}$ \\
D3 & $(0,0, \delta, 0,0,0)$ & $1 / 2 C_{33} \delta^{2}$ \\
D4 & $\left(\left(1-\delta^{2}\right)^{-1 / 3}-1,\left(1-\delta^{2}\right)^{-1 / 3}-1,\left(1-\delta^{2}\right)^{-1 / 3}-1,2 \delta\left(1-\delta^{2}\right)^{-1 / 3}, 0,0\right)$ & $2 C_{44} \delta^{2}$ \\
D5 & $\left(\left(1-\delta^{2}\right)^{-1 / 3}-1,\left(1-\delta^{2}\right)^{-1 / 3}-1,\left(1-\delta^{2}\right)^{-1 / 3}-1,0,2 \delta\left(1-\delta^{2}\right)^{-1 / 3}, 0\right)$ & $2 C_{55} \delta^{2}$ \\
D6 & $\left(\left(1-\delta^{2}\right)^{-1 / 3}-1,\left(1-\delta^{2}\right)^{-1 / 3}-1,\left(1-\delta^{2}\right)^{-1 / 3}-1,0,0,2 \delta\left(1-\delta^{2}\right)^{-1 / 3}\right)$ & $2 C_{66} \delta^{2}$ \\
D7 & $\left((1+\delta)\left(1-\delta^{2}\right)^{-1 / 3}-1,(1-\delta)\left(1-\delta^{2}\right)^{-1 / 3}-1,\left(1-\delta^{2}\right)^{-1 / 3}-1,0,0,0\right)$ & $1 / 2\left(C_{11}+C_{22}-2 C_{12}\right) \delta^{2}$ \\
D8 & $\left((1+\delta)\left(1-\delta^{2}\right)^{-1 / 3}-1,\left(1-\delta^{2}\right)^{-1 / 3}-1,(1-\delta)\left(1-\delta^{2}\right)^{-1 / 3}-1,0,0,0\right)$ & $1 / 2\left(C_{11}+C_{33}-2 C_{13}\right) \delta^{2}$ \\
D9 & $\left(\left(1-\delta^{2}\right)^{-1 / 3}-1,(1+\delta)\left(1-\delta^{2}\right)^{-1 / 3}-1,(1-\delta)\left(1-\delta^{2}\right)^{-1 / 3}-1,0,0,0\right)$ & $1 / 2\left(C_{22}+C_{33}-2 C_{23}\right) \delta^{2}$ \\
\hline
\end{tabular}

\section{Results and Discussion}

\subsection{Structural Properties}

Table 2 gives the calculated equilibrium lattice constants and atomic Wyckoff positions of B19 $\mathrm{TiAl}$ in this work. From the selected-area electron diffraction patterns, Abe et al. [2] measured the lattice parameters of B19 TiAl to be $a=4.5 \AA, b=2.8 \AA$, and $c=4.9 \AA$. Ducher et al. [5] estimated the lattice parameters of B19 TiAl to be $a=4.65 \AA, b=2.828 \AA$, and $c=4.94 \AA$, in the unit cell of which $\mathrm{Ti}$ and $\mathrm{Al}$ atoms occupy the respective Wyckoff positions $(1 / 4,0,5 / 6)$ and $(1 / 4,1 / 2$, 1/3). Song et al. [7] measured the lattice parameters of B19 phase to be $a=4.64 \AA, b=2.90 \AA$, and $c=5.10 \AA$. From high-energy X-ray diffraction patterns, Schmoelzer et al. [6] measured the lattice parameters of B19 phase to be $a=4.65 \AA, b=2.93 \AA$, and $c=4.95 \AA$. These experimental data are also given in Table 2 for comparison. It is found that the maximum deviations of the calculated equilibrium lattice constants $a, b$, and c correspond to $2.94 \%, 2.29 \%$, and $4.03 \%$ as compared with the respective experimental values. Furthermore, the calculated equilibrium atomic positions are in agreement with the respective experimental values [5]. These indicate that our calculated results agree 
basically with the available experimental results. Moreover, the formation energy $E_{\mathrm{f}}$ of $\mathrm{B} 19 \mathrm{TiAl}$ is defined as $E_{\mathrm{f}}=[E(\mathrm{TiAl})-2 E(\mathrm{Ti})-2 E(\mathrm{Al})] / 4$, where $E(\mathrm{TiAl})$ is the total energy of the whole unit cell, i.e., per 4 atoms, and $E(\mathrm{Ti})$ and $E(\mathrm{Al})$ correspond to the total energy per atom of $\mathrm{Ti}$ and $\mathrm{Al}$ elements in their standard states. The calculated formation energy is $-0.416 \mathrm{eV} /$ atom, indicating that $\mathrm{B} 19 \mathrm{TiAl}$ is energetically stable.

Table 2. The equilibrium lattice constants $(a, b, c)$ and atomic Wyckoff positions $(x, y, z)$ of B19 TiAl.

\begin{tabular}{cccccccc}
\hline Method & $\boldsymbol{a}(\AA)$ & $\boldsymbol{b}(\AA)$ & $\boldsymbol{c}(\AA)$ & Atoms & $\boldsymbol{x}$ & $\boldsymbol{y}$ & $\boldsymbol{z}$ \\
\hline This work & 4.632 & 2.863 & 4.895 & $\mathrm{Ti}$ & $1 / 4$ & 0 & 0.8398 \\
& & & & $\mathrm{Al}$ & $1 / 4$ & $1 / 2$ & 0.3392 \\
Exp. [2] & 4.50 & 2.80 & 4.90 & & & & \\
Exp. [6] & 4.65 & 2.93 & 4.95 & & & & \\
Exp. [5] & 4.65 & 2.828 & 4.94 & $\mathrm{Ti}$ & $1 / 4$ & 0 & $5 / 6$ \\
& & & & $\mathrm{Al}$ & $1 / 4$ & $1 / 2$ & $1 / 3$ \\
Exp. [7] & 4.64 & 2.90 & 5.10 & & & & \\
\hline
\end{tabular}

\subsection{Elastic and Mechanical Properties}

The relation between the variation in energy and the tensor parameter of orthorhombic B19 TiAl for each selected strain mode is fitted with a quadratic polynomial and a cubic polynomial, respectively. The second-order coefficient in the cubic polynomial fitting is found to be in agreement with that in the quadratic one because the small strains within \pm 0.015 may avoid the influence of higher-order terms on the computed coefficient. After numerically obtaining the second-order coefficients in the quadratic polynomials, the elastic constants $C_{i j}$ can be determined using the equilibrium lattice parameters. The calculated results are listed in Table 3. Obviously, the elastic constants obtained by using two $\mathrm{ab}$ initio techniques are in basic agreement with each other. The deviations are partially due to the calculation techniques and fitting errors. Unfortunately, there are no available experimental and theoretical values to check our calculated results. The obtained results in the present work could provide a useful reference for future studies. Moreover, an orthorhombic crystal will be mechanically unstable until its elastic constants satisfy the following necessary and sufficient conditions [14]:

$$
\begin{gathered}
C_{11}>0, \quad C_{11} C_{22}-C_{12}^{2}>0, \\
C_{11} C_{22} C_{33}+2 C_{12} C_{13} C_{23}-C_{11} C_{23}^{2}-C_{22} C_{13}^{2}-C_{33} C_{12}^{2}>0 \\
C_{44}>0, \quad C_{55}>0, \quad C_{66}>0 .
\end{gathered}
$$

Table 3. The elastic stiffness $\left(C_{i j}\right.$ in GPa) and compliance $\left(S_{i j}\right.$ in $\left.\mathrm{GPa}^{-1}\right)$ constants of B19 TiAl.

\begin{tabular}{ccccc}
\hline Method & \multicolumn{2}{c}{ EDM } & \multicolumn{2}{c}{ SSM } \\
\hline$i j$ & $C_{i j}$ & $S_{i j}$ & $C_{i j}$ & $S_{i j}$ \\
11 & 206.58 & 0.00560 & 206.90 & 0.00553 \\
22 & 191.33 & 0.00684 & 193.56 & 0.00660 \\
33 & 216.70 & 0.00556 & 218.56 & 0.00544 \\
44 & 53.16 & 0.01881 & 56.15 & 0.01781 \\
55 & 55.37 & 0.01806 & 54.17 & 0.01846 \\
66 & 73.08 & 0.01368 & 74.66 & 0.01339 \\
12 & 70.73 & -0.00183 & 68.03 & -0.00170 \\
13 & 48.15 & -0.00055 & 47.40 & -0.00058 \\
23 & 82.07 & -0.00218 & 79.65 & -0.00204 \\
\hline
\end{tabular}

It is clear that the orthorhombic B19 TiAl is mechanically stable in terms of our calculated elastic constants.

From the single crystal elastic constants data, the polycrystalline bulk modulus $B$ and shear modulus $G$ can be usually calculated by using the Voigt approximation [39] and the Reuss 
approximation [40] methods. For an orthorhombic crystal, the Voigt bulk $\left(B_{\mathrm{V}}\right)$ and shear $\left(G_{\mathrm{V}}\right)$ moduli are given by

$$
\begin{gathered}
B_{\mathrm{V}}=\frac{1}{9}\left(C_{11}+C_{22}+C_{33}+2 C_{12}+2 C_{13}+2 C_{23}\right), \\
G_{\mathrm{V}}=\frac{1}{15}\left(C_{11}+C_{22}+C_{33}+3 C_{44}+3 C_{55}+3 C_{66}-C_{12}-C_{13}-C_{23}\right) .
\end{gathered}
$$

The Reuss bulk $\left(B_{R}\right)$ and shear $\left(G_{R}\right)$ moduli are given by

$$
G_{R}=\frac{B_{R}=\frac{1}{\left(S_{11}+S_{22}+S_{33}\right)+2\left(S_{12}+S_{13}+S_{23}\right)},}{4\left(S_{11}+S_{22}+S_{33}\right)-4\left(S_{12}+S_{13}+S_{23}\right)+3\left(S_{44}+S_{55}+S_{66}\right)},
$$

where the $S_{i j}\left(=C_{i j}{ }^{-1}\right)$ are the elastic compliance constants. Hill [41] proposed that the effective bulk $(B)$ and shear $(G)$ moduli should be the arithmetic averages of the Voigt and Reuss bulk $\left(B_{\mathrm{V}}, B_{\mathrm{R}}\right)$ and shear $\left(G_{\mathrm{V}}, G_{\mathrm{R}}\right)$ moduli in practice, namely $B=\left(B_{\mathrm{V}}+B_{\mathrm{R}}\right) / 2$ and $G=\left(G_{\mathrm{V}}+G_{\mathrm{R}}\right) / 2$. Further, the Young's modulus $E$ and Poisson's ratio $v$ can be given by

$$
\begin{gathered}
E=\frac{9 B G}{3 B+G} \\
v=\frac{3 B-2 G}{2(3 B+G)} .
\end{gathered}
$$

Table 4 gives the calculated results of the polycrystalline bulk modulus $B$, shear modulus $G$, Young's modulus $E$, and Poisson ratio $v$ of orthorhombic B19 TiAl. The $B$ measures the resistance of a material to a volume change. The $G$ measures the resistance of a material to a shape change. The $E$ is defined as the ratio of tensile stress to tensile strain and often used to provide a measure of the stiffness of the solid. The larger the value of $E$, the stiffer the material. The calculated values of $B, G$, and $E$ of B19 TiAl from the EDM are lower $0.13 \%, 10.84 \%$, and $9.01 \%$ lower than the corresponding theoretical values of $\gamma$-TiAl $(113,70.6$, and $175 \mathrm{GPa}$ ) and $1.87 \%, 15.16 \%$, and $12.98 \%$ lower than the corresponding theoretical values of $\alpha_{2}-\mathrm{Ti}_{3} \mathrm{Al}(115,74.2$, and $183 \mathrm{GPa})$ [42]. These indicate that the plastic deformation of $\mathrm{B} 19 \mathrm{TiAl}$ is easier and its compliancy higher than $\gamma$-TiAl and $\alpha_{2}-\mathrm{Ti}_{3} \mathrm{Al}$.

Table 4. The Voigt, Reuss, and Hill bulk $\left(B_{\mathrm{V}}, B_{\mathrm{R}}, B\right.$ in $\left.\mathrm{GPa}\right)$ and shear $\left(G_{\mathrm{V}}, G_{\mathrm{R}}, G\right.$ in GPa) moduli, Young's modulus ( $E$ in GPa) and Poisson ratio $(v)$ of polycrystalline B19 TiAl.

\begin{tabular}{ccccccccc}
\hline Method & $\boldsymbol{B}_{\mathbf{V}}$ & $\boldsymbol{B}_{\mathbf{R}}$ & $\boldsymbol{B}$ & $\boldsymbol{G}_{\mathbf{V}}$ & $\boldsymbol{G}_{\mathbf{R}}$ & $\boldsymbol{G}$ & $\boldsymbol{E}$ & $\boldsymbol{v}$ \\
\hline EDM & 112.95 & 112.75 & 112.85 & 63.90 & 62.00 & 62.95 & 159.24 & 0.265 \\
SSM & 112.13 & 111.92 & 112.02 & 65.26 & 63.41 & 64.34 & 161.99 & 0.259 \\
\hline
\end{tabular}

As mentioned in Section 1, Pugh's ratio $(G / B)$, Cauchy pressure, and Poisson's ratio can allow us to know the ductile/brittle nature of a material. According to the Pugh's ratio [11], a material behaves in a ductile manner if $G / B<0.5$; otherwise, it should be brittle. The $G / B$ ratios of 0.558 and 0.574 can be obtained from two ab initio techniques for B19 TiAl. It is clear that both $G / B$ ratios are larger than 0.5. The calculated $G / B$ ratios of $B 19 \mathrm{TiAl}$ from the EDM is $10.03 \%$ and $14.18 \%$ lower than the theoretical data of $\gamma$-TiAl (0.62) and $\alpha_{2}-\mathrm{Ti}_{3} \mathrm{Al}(0.65)$ [42], respectively, indicating that $\mathrm{B} 19 \mathrm{TiAl}$ is more ductile than $\gamma$-TiAl and $\alpha_{2}-\mathrm{Ti}_{3} \mathrm{Al}$. Experimentally, Appel et al. [3,8-10] have measured the mechanical properties of $\gamma$-TiAl-based intermetallics with the microstructure containing B19 phase and found that the existence of B19 structure can give the intermetallics relatively high tensile ductility. According to Pettifor's rule [12], a material has more metallic (angular) bonds and thus is more ductile (brittle) if it has a larger positive (negative) Cauchy pressure. For the orthorhombic system, the Cauchy pressures can be defined as $C_{23}-C_{44}$ for the (100) plane, $C_{13}-C_{55}$ for the (010) plane, and $C_{12}-C_{66}$ for the (001) plane. Based on the single crystal elastic constants obtained from the two techniques, the calculated values are $28.91 \mathrm{GPa}$ and $23.50 \mathrm{GPa}$ for the pressure $\left(C_{23}-C_{44}\right),-7.22 \mathrm{GPa}$ and $-6.77 \mathrm{GPa}$ for the pressure $\left(C_{13}-C_{55}\right)$, and $-2.34 \mathrm{GPa}$ and $-6.63 \mathrm{GPa}$ for the pressure $\left(C_{12}-C_{66}\right)$ for B19 TiAl. The results 
indicate that the bonding in the (100) plane have a strong metallic character, whereas those in the (010) and (010) planes have an angular character. According to Frantsevich's rule [13], a material is brittle if its Poisson's ratio is less than 1/3, otherwise the material is ductile. The Poisson's ratios of 0.265 and 0.259 can be obtained from the two techniques for B19 TiAl. It is clear that both of the Poisson's ratios are also less than 1/3. From the above, it can be clearly shown that B19 TiAl is brittle in nature.

The elastic constants $C_{11}, C_{22}$, and $C_{33}$ and $C_{44}, C_{55}, C_{66}, C_{12}, C_{13}$, and $C_{23}$ are closely related to the elasticity of length and shape, respectively. As shown in Table 2, the elastic constant $C_{11}$ is larger than $C_{22}$ and smaller than $C_{33}$ for $\mathrm{B} 19 \mathrm{TiAl}$, implying the anisotropy of its elasticity. The elastic anisotropy can be described by the bulk modulus $\left(B_{a}, B_{b}\right.$, and $\left.B_{c}\right)$ along the axes $a, b$, and $c$ [34]. The axial bulk modulus are given by

$$
\begin{gathered}
B_{a}=a \frac{d P}{d a}=\frac{\chi}{1+\alpha+\beta}, \\
B_{b}=b \frac{d P}{d b}=\frac{B_{a}}{\alpha}, \\
B_{c}=c \frac{d P}{d c}=\frac{B_{a}}{\beta}, \\
\chi=C_{11}+2 C_{12} \alpha+C_{22} \alpha+2 C_{13} \beta+C_{33} \beta^{2}+2 C_{23} \alpha \beta, \\
\alpha=\frac{\left(C_{11}-C_{12}\right)\left(C_{33}-C_{13}\right)-\left(C_{23}-C_{13}\right)\left(C_{11}-C_{13}\right)}{\left(C_{33}-C_{13}\right)\left(C_{22}-C_{12}\right)-\left(C_{13}-C_{23}\right)\left(C_{12}-C_{23}\right)}, \\
\beta=\frac{\left(C_{22}-C_{12}\right)\left(C_{11}-C_{13}\right)-\left(C_{11}-C_{12}\left(C_{23}-C_{12}\right)\right.}{\left(C_{22}-C_{12}\right)\left(C_{33}-C_{13}\right)-\left(C_{12}-C_{23}\right)\left(C_{13}-C_{23}\right)} .
\end{gathered}
$$

With respect to the $b$ axis, the anisotropies of the bulk modulus along the axes $a$ and $c$ are defined as

$$
\begin{aligned}
& A_{a}=\frac{B_{a}}{B_{b}}=\alpha, \\
& A_{c}=\frac{B_{c}}{B_{b}}=\frac{\alpha}{\beta} .
\end{aligned}
$$

The calculated values of $B_{a}, B_{b}, B_{c}$, and $A_{a}, A_{c}$ are listed in Table 5. It is clear that the value of $B_{a}$ is significantly smaller than those of $B_{b}$ and $B_{c}$ for two different ab initio techniques, whereas in both cases the value of $B_{b}$ is very close to that of $B_{c}$. The results indicate that the compressibility along the $a$ axis is the largest whereas those along the axes $b$ and $c$ are very close to each other. Additionally, it is found that the value of $A_{a}$ is significantly smaller than one, whereas that of $A_{c}$ is very close to one. For an isotropic crystal the values of $A_{a}$ and $A_{c}$ must be one, while any departures from one measure the anisotropic degree of the crystal. Hence, B19 TiAl is an elastic anisotropic crystal.

Table 5. The bulk modulus along the axes $a, b, c\left(B_{a}, B_{b}, B_{c}\right.$ in GPa $)$ and anisotropic factors $\left(A_{a}, A_{c}\right)$ of B19 TiAl.

\begin{tabular}{llllll}
\hline \multirow{2}{*}{ Method } & \multicolumn{2}{l}{ Axial Bulk Modulus } & & \multicolumn{2}{l}{ Anisotropy Factor } \\
\cline { 2 - 6 } & $\boldsymbol{B}_{\boldsymbol{a}}$ & $\boldsymbol{B}_{\boldsymbol{b}}$ & $\boldsymbol{B}_{\boldsymbol{c}}$ & $\boldsymbol{A}_{\boldsymbol{a}}$ & $\boldsymbol{A}_{\boldsymbol{c}}$ \\
\hline EDM & 311.15 & 354.10 & 353.23 & 0.879 & 0.998 \\
SSM & 308.25 & 349.40 & 353.49 & 0.882 & 1.012 \\
\hline
\end{tabular}

In addition to the axial bulk modulus, the shear anisotropy factors $\left(A_{\{100\}}, A_{\{010\}}\right.$, and $\left.A_{\{001\}}\right)$ in the atomic bonding for $\{100\},\{010\}$, and $\{001\}$ planes can be calculated in terms of the elastic constants [34], which are given by

$$
\begin{aligned}
& A_{\{100\}}=\frac{4 C_{44}}{C_{11}+C_{33}-2 C_{13}}, \\
& A_{\{010\}}=\frac{4 C_{55}}{C_{22}+C_{33}-2 C_{23}}, \\
& A_{\{001\}}=\frac{4 C_{66}}{C_{11}+C_{22}-2 C_{12}} .
\end{aligned}
$$

For an isotropic crystal the values of $A_{\{100\}}, A_{\{010\}}$, and $A_{\{001\}}$ must be one, while any departures from one are a measure of the anisotropic degree of the crystal. Chung and Buessem [43] have 
introduced the percent anisotropy factors of bulk modulus $\left(A_{B}\right)$ and shear modulus $\left(A_{G}\right)$ to measure the crystal anisotropy, which are defined as

$$
\begin{aligned}
& A_{B}=\frac{B_{\mathrm{V}}-B_{\mathrm{R}}}{B_{\mathrm{V}}+B_{\mathrm{R}}} \times 100 \%, \\
& A_{G}=\frac{G_{\mathrm{V}}-G_{\mathrm{R}}}{G_{\mathrm{V}}+G_{\mathrm{R}}} \times 100 \% .
\end{aligned}
$$

Ranganathan and Ostoja-Starzewski [44] have introduced a universal anisotropy factor to measure the crystal anisotropy, which is defined as

$$
A^{\mathrm{U}}=5 \frac{\mathrm{G}_{\mathrm{V}}}{G_{\mathrm{R}}}+\frac{B_{\mathrm{V}}}{B_{\mathrm{R}}}-6
$$

For an isotropic crystal the values of $A_{B}, A_{G}$, and $A^{\mathrm{U}}$ must be zero, while any departures from zero are a measure of the anisotropic degree of the crystal. The calculated values of various anisotropy factors are listed in Table 6. It is clear that the values of $A_{\{100\}}, A_{\{010\}}$, and $A_{\{001\}}$ are all departed from one and the $A_{\{100\}}$ has the largest absolute deviation from one, which indicates that the shear anisotropy for the $\{100\}$ plane is the strongest. Additionally, it is observed that all of $A_{B}, A_{G}$, and $A^{\mathrm{U}}$ have nonzero positive values, and the value of $A_{G}$ is significantly larger than that of $A_{B}$. These show that $\mathrm{B} 19 \mathrm{TiAl}$ is elastic anisotropic and exhibits a small anisotropy of bulk modulus but a high anisotropic degree of shear modulus.

Table 6. The shear anisotropy factors $\left(A_{\{100\}}, A_{\{010\}}\right.$, and $\left.A_{\{001\}}\right)$, percent anisotropy factors of bulk $\left(A_{B}\right)$ and shear $\left(A_{G}\right)$ moduli, and universal anisotropy factor $\left(A^{\mathrm{U}}\right)$ of B19 TiAl.

\begin{tabular}{ccccccc}
\hline Method & $\boldsymbol{A}_{\{\mathbf{1 0 0 \}}}$ & $\boldsymbol{A}_{\{\mathbf{0 1 0 \}}}$ & $\boldsymbol{A}_{\{\mathbf{0 0 1 \}}}$ & $\boldsymbol{A}_{\boldsymbol{B}} \mathbf{( \% )}$ & $\boldsymbol{A}_{\boldsymbol{G}} \mathbf{( \% )}$ & $A^{\mathrm{U}}$ \\
\hline EDM & 0.650 & 0.908 & 1.140 & 0.086 & 1.508 & 0.155 \\
SSM & 0.679 & 0.857 & 1.130 & 0.095 & 1.437 & 0.148 \\
\hline
\end{tabular}

Nevertheless, these factors are not enough for the complete description of the elastic anisotropic behavior of the crystals. As a matter of fact, the orientation dependence of Young's modulus $E$ is usually employed to analyze the elastic anisotropy of the crystals. For an orthorhombic crystal, the Young's modulus in any orientation is given by [45]

$$
\frac{1}{E}=S_{11} l_{1}^{4}+\left(2 S_{12}+S_{66}\right) l_{1}^{2} l_{2}^{2}+S_{22} l_{2}^{4}+\left(2 S_{23}+S_{44}\right) l_{2}^{2} l_{3}^{2}+S_{33} l_{3}^{4}+\left(2 S_{13}+S_{55}\right) l_{1}^{2} l_{3}^{2},
$$

where $l_{1}, l_{2}$, and $l_{3}$ are direction cosines. Figure 1 shows the orientation dependent Young's modulus of B19 TiAl calculated by using the elastic compliance constants obtained from two different ab initio techniques. It is clear that the Young's modulus surface of the energy density method is overall similar in contour to that of the strain-stress method. The Young's modulus surface is a perfect spherical surface for an isotropic crystal, but are both not the case for B19 TiAl. Furthermore, the orientation dependence of Young's modulus in the $a b, b c$, and $c a$ planes are shown in Figure 2a-c, respectively. The orbit of Young's modulus in any plane should be a perfect circle for an isotropic crystal, but are also not the case for B19 TiAl. These clearly show that B19 TiAl exhibit elastic anisotropy. 

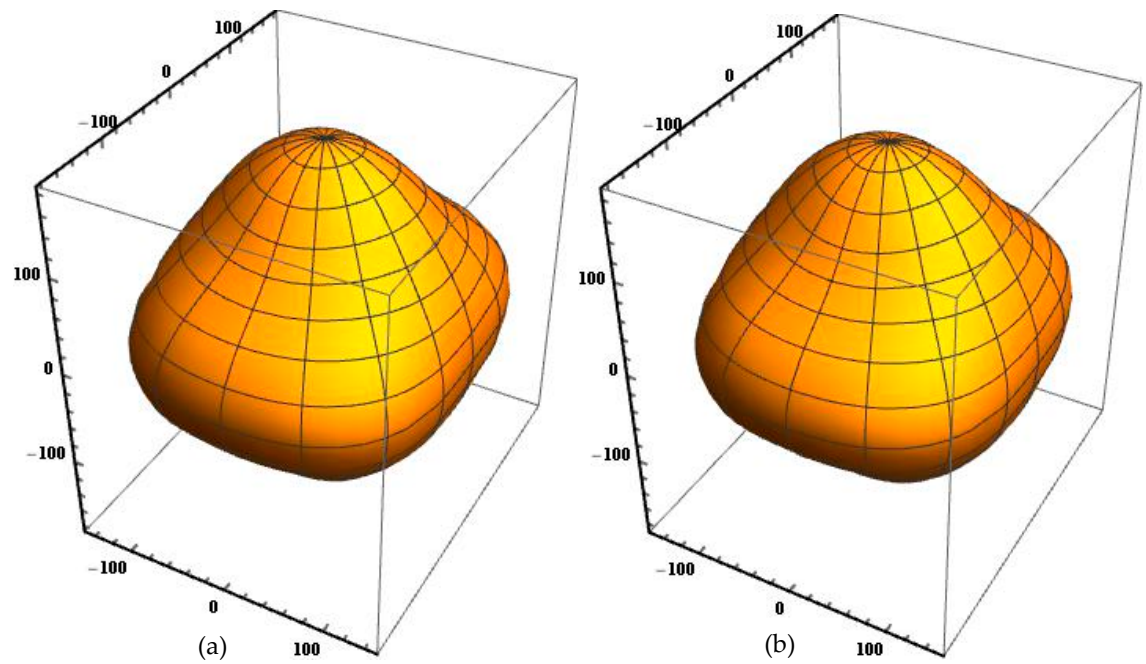

Figure 1. The orientation dependent Young's modulus of B19 TiAl using the elastic constants obtained from the energy density (a) and the strain-stress (b) methods.
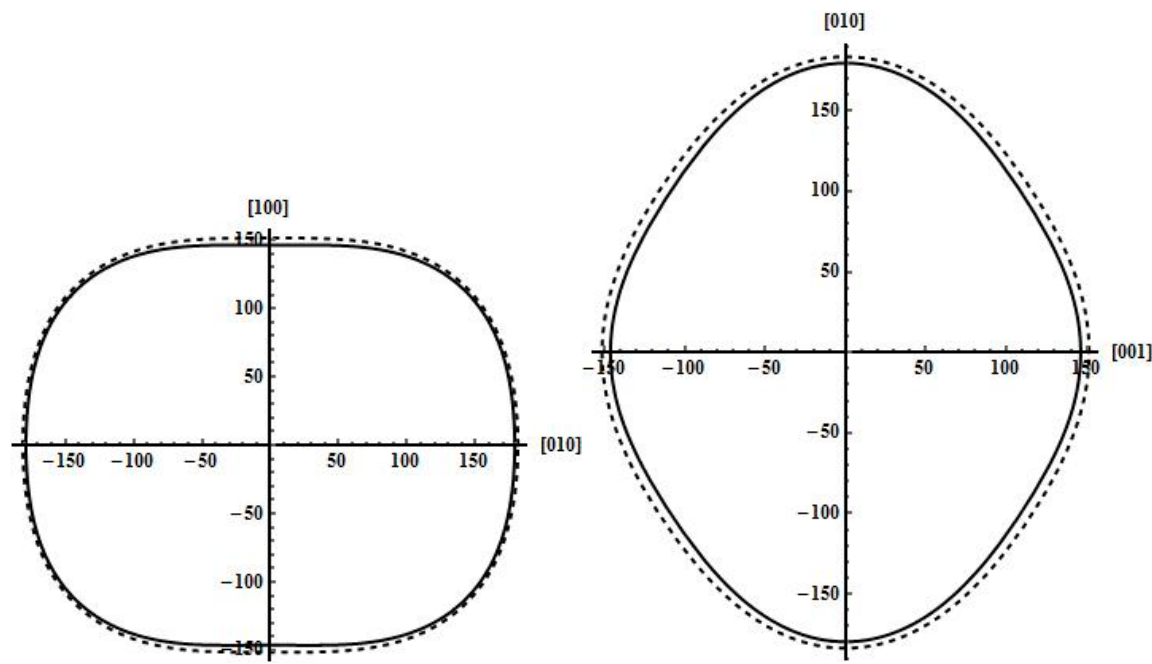

(a)

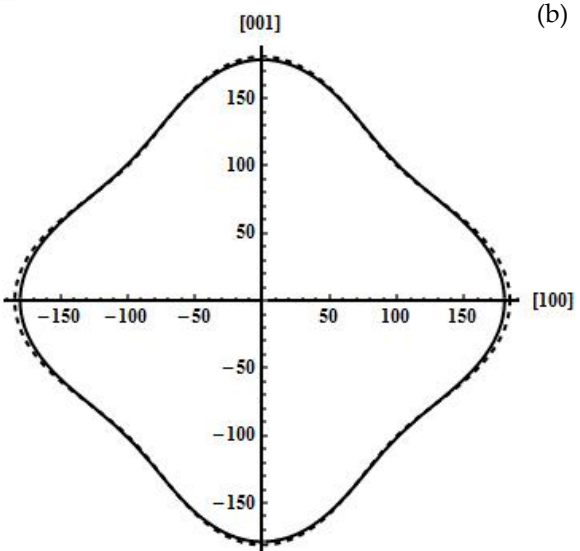

(c)

Figure 2. The orientation dependent Young's modulus of B19 TiAl in the $a b$ plane (a) with the tensile axis rotated from [100] to [010], the $b c$ plane (b) with the tensile axis rotated from [010] to [001], and the ca plane (c) with the tensile axis rotated from [001] to [100]. The solid and dashed lines represent the calculated values of the energy density and the strain-stress methods, respectively. 


\section{Conclusions}

First-principles calculations have been performed in order to predict the structural, elastic, and mechanical properties of orthorhombic B19 TiAl intermetallic compound at ground state. The calculated lattice parameters and atomic positions are in agreement with the available experimental data. The calculated formation energy shows that B19 TiAl is energetically stable. Moreover, the single crystal elastic constants are also determined by using the energy density and the strain-stress methods, respectively. The obtained elastic constants show that the orthorhombic B19 TiAl is mechanically stable. Further, the polycrystalline bulk modulus, shear modulus, Young's modulus, Poisson's ratio, Cauchy pressure, and anisotropy factors of B19 TiAl are calculated in terms of its single crystal elastic constants. From the calculated Pugh's ratio, Cauchy pressure, and Poisson's ratio, it is shown that B19 TiAl has intrinsic brittleness. A smaller Pugh's ratio of B19 TiAl than $\gamma$-TiAl and $\alpha_{2}-\mathrm{Ti}_{3} \mathrm{Al}$ indicates its relative ductility. Various anisotropy factors and the orientation dependence of Young's modulus of B19 TiAl show its anisotropy of elasticity. Because there are no available experimental values of elastic properties in the literature for $\mathrm{B} 19 \mathrm{TiAl}$, we think that the first-principles estimation is the only reasonable tool for obtaining such important information.

Acknowledgments: This project was granted financial support from National Natural Science Foundation of China (No. 51661013, 51601146).

Author Contributions: Long Wang and Huilong Liu carried out the theoretical calculations; Yufeng Wen and Lin Song analyzed the data; Long Wang wrote the paper.

Conflicts of Interest: The authors declare no conflict of interest.

\section{References}

1. Kestler, H.; Clemens, H. Production, processing and application of $\gamma(\mathrm{TiAl})$-based alloys. In Titanium and Titantium Alloys; Leyens, C., Peters, M., Eds.; Wiley: Weinheim, Germany, 2003; p. 351.

2. Abe, E.; Kumagai, T.; Nakamura, M. New ordered structure of TiAl studied by high-resolution electron microscopy. Intermetallics 1996, 4, 327-333. [CrossRef]

3. Appel, F.; Oehring, M.; Paul, J.D.H. Nano-scale design of TiAl alloys based on $\beta$-phase decomposition. Adv. Eng. Mater. 2006, 8, 371-376. [CrossRef]

4. Tanimura, M.; Inoue, Y.; Koyama, Y. Change in $\mathrm{Al}$ concentration profile related to the $\mathrm{D}_{19} \rightarrow \mathrm{L}_{0}$ structural change in Ti-Al alloys. Scr. Mater. 2001, 44, 365-373. [CrossRef]

5. Ducher, R.; Viguier, B.; Lacaze, J. Modification of the crystallographic structure of $\gamma$-TiAl alloyed with iron. Scr. Mater. 2002, 47, 307-313. [CrossRef]

6. Schmoelzer, T.; Stark, A.; Schwaighofer, E.; Lippmann, T.; Mayer, S.; Clemens, H. In situ synchrotron study of B19 phase formation in an intermetallic $\gamma$-TiAl alloys. Adv. Eng. Mater. 2012, 14, 445-448. [CrossRef]

7. Song, L.; Xu, X.J.; You, L.; Liang, Y.F.; Lin, J.P. B19 phase in Ti- $45 \mathrm{Al}-8.5 \mathrm{Nb}-0.2 \mathrm{~W}-0.2 \mathrm{~B}-0.02 \mathrm{Y}$ alloy. J. Alloys Compd. 2015, 618, 305-310. [CrossRef]

8. Appel, F.; Oehring, M.; Paul, J.D.H. A novel in situ composite structure in TiAl alloys. Mater. Sci. Eng. A 2008, 493, 232-236. [CrossRef]

9. Appel, F.; Heckel, T.K.; Christ, H.J. Electron microscope characterization of low cycle fatigue in a high-strength multiphase titanium aluminide alloy. Int. J. Fatigue 2010, 32, 792-798. [CrossRef]

10. Appel, F.; Paul, J.D.H.; Oehring, M. Phase transformations during creep of a multiphase TiAl-based alloy with a modulated microstructure. Mater. Sci. Eng. A 2009, 510-511, 342-349. [CrossRef]

11. Pugh, S.F. Relations between the elastic moduli and the plastic properties of polycrystalline pure metals. Philos. Mag. 1954, 45, 823-843. [CrossRef]

12. Pettifor, D. Theoretical predictions of structure and related properties of intermetallics. Mater. Sci. Technol. 1992, 8, 345-349. [CrossRef]

13. Frantsevich, I.N.; Voronov, F.F.; Bokuta, S.A. Elastic Constants and Elastic Moduli of Metals and Insulators Handbook; Frantsevich, I.N., Ed.; Naukova Dumka: Kiev, Ukraine, 1983; pp. 60-180.

14. Mouhat, F.; Coudert, F.X. Necessary and sufficient elastic stability conditions in various crystal systems. Phys. Rev. B 2014, 90, 224104. [CrossRef] 
15. Music, D.; Schneider, J.M. Effect of transition metal additives on electronic structure and elastic properties of TiAl and $\mathrm{Ti}_{3} \mathrm{Al}$. Phys. Rev. B 2006, 74, 174110. [CrossRef]

16. Fu, H.; Li, D.; Peng, F.; Gao, T.; Cheng, X. Structural and elastic properties of $\gamma$ TiAl under high pressure from electronic structure calculations. J. Alloys Compd. 2009, 473, 255-261. [CrossRef]

17. Wei, Y.; Zhang, Y.; Lu, G.H.; Xu, H.B. Site preference and elastic properties of $\alpha_{2}-\mathrm{Ti}_{3} \mathrm{Al}$ with oxygen impurity: a first-principles study. Int. J. Mod. Phys. B 2010, 24, 2749-2755. [CrossRef]

18. Tang, P.Y.; Tang, B.Y.; Xu, X.P. First-principles studies of typical long-period superstructures $\mathrm{Al}_{5} \mathrm{Ti}_{3}, \mathrm{~h}-\mathrm{Al}_{2} \mathrm{Ti}$ and $\mathrm{r}-\mathrm{Al}_{2} \mathrm{Ti}$ in Al-rich TiAl alloys. Comp. Mater. Sci. 2011, 50, 1467-1476. [CrossRef]

19. Tang, P.Y.; Huang, G.H.; Xie, Q.L.; Huang, X.L.; Ning, F. Elastic anisotropy and phonon focusing for tetragonal crystals: Application to $\gamma$-TiAl. Comp. Mater. Sci. 2016, 118, 117-123. [CrossRef]

20. Ghosh, P.S.; Arya, A.; Kulkarni, U.D.; Dey, G.K.; Hata, S.; Nakano, T.; Hagiharadan, K.; Nakashima, H. $\mathrm{Ab}$-initio study of long-period superstructures and anti-phase boundaries in Al-rich $\gamma$-TiAl $\left(\mathrm{L} 1_{0}\right)$-based alloys. Philos. Mag. 2014, 60, 175-178.

21. Hu, H.; Wu, X.; Wang, R.; Li, W.; Liu, Q. Phase stability, mechanical properties and electronic structure of TiAl alloying with W, Mo, Sc and Yb: First-principles study. J. Alloys Compd. 2016, 658, 689-696. [CrossRef]

22. Hu, H.; Wu, X.; Wang, R.; Jia, Z.; Li, W.; Liu, Q. Structural stability, mechanical properties and stacking fault energies of $\mathrm{TiAl}_{3}$ alloyed with $\mathrm{Zn} \mathrm{Cu}$, Ag: First-principles study. J. Alloys Compd. 2016, 666, 185-196. [CrossRef]

23. Nguyen-Manh, D.; Pettifor, D.G. Electronic structure, phase stability and elastic moduli of AB transition metal aluminides. Intermetallics 1999, 7, 1095-1106. [CrossRef]

24. Nguyen-Manh, D.; Pettifor, D.G. Origin of O-phase and pseudo-twinning in Ti-Al-Nb alloys: A first-principles study. In Gamma Titanium Aluminides; Kim, Y.-W., Dimiduk, D.M., Loretto, M.H., Eds.; TMS: Warrendale, PA, USA, 1999; pp. 175-182.

25. Holec, D.; Reddy, R.K.; Klein, T.; Clemens, H. Preferential site occupancy of alloying elements in TiAl-based phases. J. Appl. Phys. 2016, 119, 339-341. [CrossRef]

26. Blöchl, P.E. Projector augmented-wave method. Phys. Rev. B 1994, 50, 17953-17979. [CrossRef]

27. Perdew, J.P.; Burke, K.; Ernzerhof, M. Generalized gradient approximation made simple. Phys. Rev. Lett. 1996, 77, 3865-3868. [CrossRef] [PubMed]

28. Perdew, J.P.; Burke, K.; Ernzerhof, M. Generalized gradient approximation made simple [Phys. Rev. Lett. 77 3865 (1996)]. Phys. Rev. Lett. 1997, 78, 1396. [CrossRef]

29. Kresse, G.; Furthmüller, J. Efficiency of ab-initio total energy calculations for metals and semiconductors using a plane-wave basis set. Comput. Mater. Sci. 1996, 6, 15-150. [CrossRef]

30. Kresse, G.; Furthmüller, J. Efficient iterative schemes for ab initio total-energy calculations using a plane-wave basis set. Phys. Rev. B 1996, 54, 11169-11186. [CrossRef]

31. Kresse, G.; Joubert, D. From ultrasoft pseudopotentials to the projector augmented-wave method. Phys. Rev. B 1999, 59, 1758-1775. [CrossRef]

32. Monkhorst, H.J.; Pack, J.D. Special points for Brillouin-zone integrations. Phys. Rev. B 1976, 13, 5188-5192. [CrossRef]

33. Wallace, D.C. Thermodynamics of Crystals; Wiley: New York, NY, USA, 1972.

34. Ravindran, P.; Fast, L.; Korzhavyi, P.A.; Johansson, B.; Wills, J.; Eriksson, O. Density functional theory for calculation of elastic properties of orthorhombic crystals: Application to TiSi 2 . J. Appl. Phys. 1998, 84, 4891-4904. [CrossRef]

35. Li, S.; Ju, X.; Wan, C. Theoretical studies of elastic properties of orthorhombic $\mathrm{LiBH}_{4}$. Comput. Mater. Sci. 2014, 81, 378-385. [CrossRef]

36. Le Page, Y.; Saxe, P. Symmetry-general least-squares extraction of elastic data for strained materials from ab initio calculations of stress. Phys. Rev. B 2002, 65, 104104-104117. [CrossRef]

37. Wu, X.; Vanderbilt, D.; Hamann, D.R. Systematic treatment isplacements, and electric fields in density-functional perturbation theory. Phys. Rev. B 2005, 72, 035105-035117. [CrossRef]

38. Erba, A. The internal-strain tensor of crystals for nuclear-relaxed elastic and piezoelectric constants: On the full exploitation of its symmetry features. Phys. Chem. Chem. Phys. 2016, 18, 13984-13992. [CrossRef] [PubMed]

39. Voigt, W. Lehrbuch der Kristallphysik; Vieweg + Teubner Verlag: Leipzig, Germany, 1928. 
40. Reuss, A.; Angew, Z. Berechnung del fliessgrenze von mischkristallen auf grund der plastizitatbedingung for einkristalle. Math. Mech. 1929, 9, 49-58.

41. Hill, R. The elastic behaviour of a crystalline aggregate. Proc. Phys. Soc. A 1953, 65, 349-354. [CrossRef]

42. Chen, S.; Liang, C.P.; Gong, H.R. Structural stability, mechanical property and elastic anisotropy of TiAl-H system. Int. J. Hydrog. Energy 2012, 37, 2676-2684. [CrossRef]

43. Chung, D.H.; Buessem, W.R. The elastic anisotropy of crystals. J. Appl. Phys. 1967, 38, 2010-2012. [CrossRef]

44. Ranganathan, S.I.; Ostoja-Starzewski, M. Universal elastic anisotropy index. Phys. Rev. Lett. 2008, 101, 055504. [CrossRef] [PubMed]

45. Nye, J.F. Physical Properties of Crystals; Oxford University Press: Oxford, UK, 1985.

(C) 2017 by the authors; licensee MDPI, Basel, Switzerland. This article is an open access article distributed under the terms and conditions of the Creative Commons Attribution (CC BY) license (http:/ / creativecommons.org/licenses/by/4.0/). 